\title{
Antenatal Diagnosis of Chiari Type II Malformation: A Case Report and a Review of the Literature
}

\author{
Meryem Belmajdoub*, Hind Adadi, Sofia Jayi, Fatima Zehra Fdili Alaoui, Hekmat Chaara, Moulay Abdelilah Melhouf \\ Department of Obstetrics and Gynaecology, Hassan II University Hospital in Fez, Morocco
}

\begin{tabular}{ll}
\hline DOI: $10.36348 /$ SIJOG.2019.v02i10.004 & | Received: 01.10.2019| Accepted: 08.10.2019| Published: 30.10 .2019
\end{tabular}

*Corresponding author: Meryem Belmajdoub

\section{Abstract}

The antenatal diagnosis of isolated abnormalities of the posterior fossa is possible thanks to ultrasound screening and antenatal MRI, which are currently of excellent quality. The Arnold Chiari type 2 malformation is a rare congenital malformation of the cerebellum, it results from the normal growth of nerve elements in a posterior fossa too small. It is always associated with myelomeningocele. We report the case of an antenatal discovery, at 36 weeks of amenorrhea, of a Chiari type II malformation associated with a myelomeningocele. Through this case and a review of literature we will highlight the contribution of different methods of antenatal imaging in the diagnosis of this rare malformation.

Keywords: Antenatal Diagnosis, antenatal MRI, cerebellum, myelomeningocele.

Copyright @ 2019: This is an open-access article distributed under the terms of the Creative Commons Attribution license which permits unrestricted use, distribution, and reproduction in any medium for non-commercial use (NonCommercial, or CC-BY-NC) provided the original author and source are credited.

\section{INTRODUCTION}

The antenatal diagnosis of isolated abnormalities of the posterior fossa is possible thanks to ultrasound screening and antenatal MRI, which are currently of excellent quality [1]. The Arnold Chiari type 2 malformation is a rare congenital malformation of the cerebellum, it results from the normal growth of nerve elements in a posterior fossa too small. It is always associated with myelomeningocele [2]. We report the case of an antenatal discovery, at 36 weeks of amenorrhea, of a Chiari type II malformation associated with a myelomeningocele. Through this case and a review of literature we will highlight the contribution of different methods of antenatal imaging in the diagnosis of this rare malformation.

\section{CASE REPORT}

Mrs $\mathrm{OH}$ aged 30, primigest, without medical or surgical history, without notion of consanguineous marriage who consulted in our training at 36 SA having benefited from an obstetric ultrasound which objectified: evolutionary mono-fetal pregnancy, correct biometry compared to gestational age with ventriculomegaly and atrophic cerebral fossa posterior (Figure-1). A fetal MRI was performed and showed the presence of tri ventricular hydrocephalus with absence of individualization of the 4th ventricle and a small FCP with tonsillar hernia and vermis below the occipital fossa (Figure-2), we note also the presence of a sacred spina bifida without individualizable liquid mass opposite (Figure-2). No other malformation was detected, the diagnosis of a Chiari type II malformation associated with spina bifida was retained. At 40 weeks the patient received a caesarean section for acute fetal sulfur giving birth to a newborn male, weighing $3100 \mathrm{~g}$, with a respiratory distress of $5 / 10$, clinical examination revealed the presence of a ruptured myelomeningocele with exposure of the medullary plaque (Figure-3), the bulging anterior fontanelle without motor deficit of the 2 lower limbs, trans sonography fontanelle made: triventricular hydrocephalus. At day 1, the baby benefited by pediatric surgeons from a ventriculoperitoneal shunt and then diedat $d+2$ in a table of acute respiratory distress. 


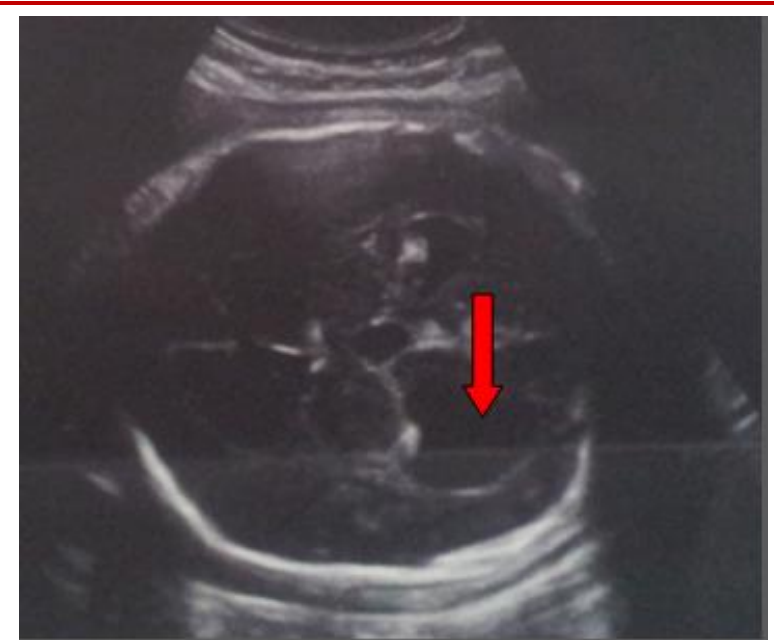

Fig-1: antenatal ultrasound (cross section) Triventricular dilatation with triangular aspect of the posterior horn of the lateral ventricle

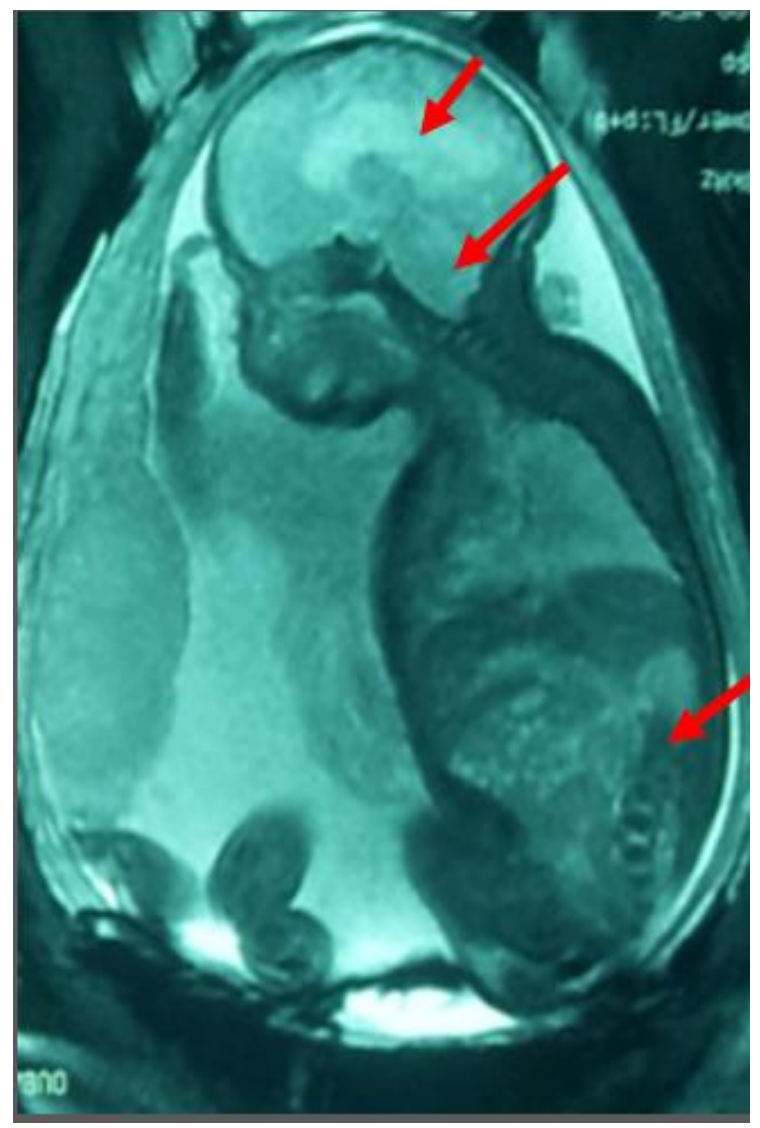

Fig-2: Fetal MRI (sagittal section) Hydrocephalus with small volume FCP spina bifida without fluid collection

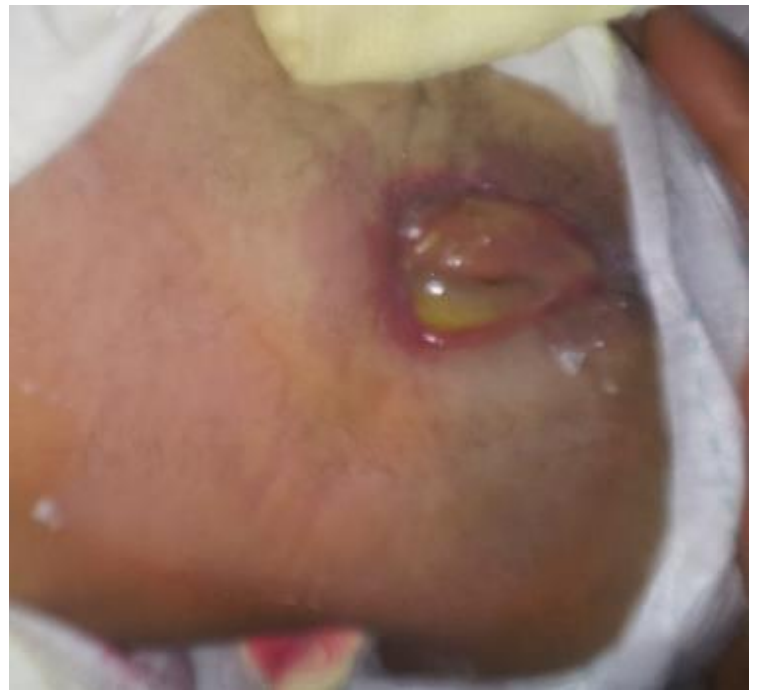

Fig-3: Clinical aspect of a ruptured myelomeningocele at birth

\section{DISCUSSION}

The Arnold-Chiari malformation is a rare congenital malformation of the cerebellum. It affects about $0.01 \%$ of the pediatric population [1-3]. There are 4 types: types I and II are the most frequently encountered, types III and IV are often lethal. The Chiari type 2 malformation results from the normal growth of nerve elements in a posterior fossa that is too small [2]. Its Incidence is 0.4 / 1000 births [3], It is characterized by: An ectopia of the lower cerebellar vermis in the upper part of the cervical canal through the occipital foramen with a with herniation of the bulb and forth ventricle [1]. It is always associated with spina bifida with lumbosacral myelomeningocele [2]. In addition, because of the poor circulation of CSF, the majority of these patients suffer from hydrocephalus. Antenatal ultrasound is the imaging of choice in the antenatal screening of these brain malformations and in particular the posterior fossa, on a coronal section, the triangular dilatation and quadrilateral angular form of the posterior horn of the lateral ventricle are 2 specific signs of the Chiari type 2 malformation, but the antenatal MRI is the key examination for the positive diagnosis but also for the assessment of lesions in search of other associated malformations thus making it possible to guide the obstetric decision [4]. The diagnosis is made on the MRI in sagittal section by showing: The cerebellar tonsils which close the foramen magnum, The large cistern which is always absent, The 4th ventricle which is hardly visible or absent, The posterior fossa is small by associated osteomeningeal dysplasia [4-5]. Its symptoms depend on the level of movement of the cerebellum and the pressure it exerts on adjacent nerve structures including the brainstem and cranial nerves [5]. Hydrocephalus can also cause or worsen symptoms. These symptoms include difficulty in swallowing, cyanosis during feeding, regurgitation through the nose, periods of apnea, weakness of the muscles of the face and upper limbs, nystagmus, weakness or absence of crying, torticollis [6]. Its evolution is serious because of severe 
damage to the cranial nerves and the medulla oblongata resulting in death by stopping the respiratory control [6]. The treatment is primarily surgical. It aims to stop the growth of the medullary cavity and to reduce the neurological signs. The purpose of the surgery is to create a space to decompress the cervical area and allow normal CSF circulation again at this location [7].

\section{CONCLUSION}

The malformation of Arnold-Chiari is rare. His diagnosis is essentially based on MRI, which will also aim to find other associated anomalies and to guide the obstetric decision.

Conflicts of Interest: The authors do not declare any conflict of interest.

Authors Contributions: All authors contributed to the critical review of the work. All also read and approved the final version of the manuscript.

\section{REFERENCES}

1. Novegno, F., Caldarelli, M., Massa, A., Chieffo, D., Massimi, L., Pettorini, B., ... \& Di Rocco, C. (2008). The natural history of the Chiari Type I anomaly. Journal of Neurosurgery: Pediatrics, 2(3), 179-187.
2. Aitken, L. A., Lindan, C. E., Sidney, S., Gupta, N., Barkovich, A. J., Sorel, M., \& Wu, Y. W. (2009). Chiari type I malformation in a pediatric population. Pediatric neurology, 40(6), 449-454.

3. Ando, K., Ishikura, R., Ogawa, M., Shakudo, M., Tanaka, H., Minagawa, K., ... \& Hirota, S. (2007). MRI tight posterior fossa sign for prenatal diagnosis of Chiari type II malformation. Neuroradiology, 49(12), 1033 1039.

4. Tsai, T., Bookstein, F. L., Levey, E., \& Kinsman, S. L. (2002). Chiari-II malformation: a biometric analysis. European Journal of Pediatric Surgery, 12(S 1), 12-18.

5. Appasamy, M., Roberts, D., Pilling, D., \& Buxton, N. (2006). Antenatal ultrasound and magnetic resonance imaging in localizing the level of lesion in spina bifida and correlation with postnatal outcome. Ultrasound in Obstetrics and Gynecology: The Official Journal of the International Society of Ultrasound in Obstetrics and Gynecology, 27(5), 530-536.

6. Nicolaides, K. H., Gabbe, S. G., Campbell, S., \& Guidetti, R. (1986). Ultrasound screening for spina bifida: cranial and cerebellar signs. The Lancet, 328(8498), 72-74.

7. Pooh, R. K., Maeda, K., \& Pooh, K. H. (2003). An atlas of fetal central nervous system disease: diagnosis and management. Taylor \& Francis. 